\title{
MSRE-HTPrimer: a high-throughput and genome-wide primer design pipeline optimized for epigenetic research
}

Ram Vinay Pandey ${ }^{1,2}$, Walter Pulverer ${ }^{1}$, Rainer Kallmeyer ${ }^{1}$, Gabriel Beikircher ${ }^{1}$, Stephan Pabinger ${ }^{1}$, Albert Kriegner $^{1}$ and Andreas Weinhäusel ${ }^{1 *}$

\begin{abstract}
Background: Methylation-sensitive restriction enzymes - polymerase chain reaction (MSRE-PCR) has been used in epigenetic research to identify genome-wide and gene-specific DNA methylation. Currently, epigenome-wide discovery studies provide many candidate regions for which the MSREqPCR approach can be very effective to confirm the findings. MSREqPCR provides high multiplexing capabilities also when starting with limited amount of DNA-like cfDNA to validate many targets in a time- and cost-effective manner. Multiplex design is challenging and cumbersome to define specific primers in an effective manner, and no suitable software tools are freely available for high-throughput primer design in a time-effective manner and to automatically annotate the resulting primers with known SNPs, CpG, repeats, and RefSeq genes. Therefore a robust, powerful, high-throughput, optimized, and methylation-specific primer design tool with great accuracy will be very useful.
\end{abstract}

Results: We have developed a novel pipeline, called MSRE-HTPrimer, to design MSRE-PCR and genomic PCR primers pairs in a very efficient manner and with high success rate. First, our pipeline designs all possible PCR primer pairs and oligos, followed by filtering for SNPs loci and repeat regions. Next, each primer pair is annotated with the number of cut sites in primers and amplicons, upstream and downstream genes, and CpG islands loci. Finally, MSRE-HTPrimer selects resulting primer pairs for all target sequences based on a custom quality matrix defined by the user. MSRE-HTPrimer produces a table for all resulting primer pairs as well as a custom track in GTF file format for each target sequence to visualize it in UCSC genome browser.

Conclusions: MSRE-HTPrimer, based on Primer3, is a high-throughput pipeline and has no limitation on the number and size of target sequences for primer design and provides full flexibility to customize it for specific requirements. It is a standalone web-based pipeline, which is fully configured within a virtual machine and thus can be readily used without any configuration. We have experimentally validated primer pairs designed by our pipeline and shown a very high success rate of primer pairs: out of 190 primer pairs, $71 \%$ could be successfully validated. The MSRE-HTPrimer software is freely available from http://sourceforge.net/p/msrehtprimer/wiki/Virtual_Machine/ as a virtual machine.

Keywords: PCR, Primer design, DNA methylation, Methylation sensitive restriction enzyme, High-throughput, CpG islands, MSRE-PCR

\footnotetext{
* Correspondence: andreas.weinhaeusel@ait.ac.at

${ }^{1}$ Health \& Environment Department, Molecular Diagnostics, AlT_Austrian

Institute of Technology GmbH, Vienna, Austria

Full list of author information is available at the end of the article
} 


\section{Background}

DNA methylation is a chemically stable key player in epigenetics and heritable over many generations of cell divisions [1]. It is the only known endogenous modification of DNA in mammals and refers to the enzymatic, post synthetic addition of a methyl group to the carbon 5 position of the cytosine ring [2]. Bisulfite-based methods and methylation-sensitive restriction enzyme-based PCR (MSREqPCR) methods have been used for detection of DNA methylation. In bisulfite-based methods, the genomic DNA is first treated with bisulfite, thus converting non-methylated cytosine to uracil by deamination while methylated cytosine is protected. However, this procedure has several limitations, including the inability to discriminate between 5-methylcytosine and 5-hydroxymethylcyosine, the degradation of DNA during bisulfite treatment, high experimental time, and the possibility of incomplete conversion under not ideal reaction parameters (Table 1).

MSREqPCR in contrast to bisulfite PCR can be used for the rapid, simultaneous detection of DNA methylation in multiple fragments when only a limited amount of DNA is available. It is a procedure based on the fact that digestion of genomic DNA with methylation-sensitive restriction enzymes is blocked when methylated. Best suited for that analyses targeting 5-methylcytosine are enzymes, which contain $\mathrm{CpG}$ motifs in their recognition sequence such as AciI, Hin6I, HpaII, and HpyCH4IV $[3,4]$. MSREqPCR-based method allows for a high level of multiplexing with manageable efforts regarding assay optimization, and only a few nanograms of DNA (10$20 \mathrm{ng}$ ) are needed per 100 assays [5]. However, this method has some limitations such as (1) all CpGs without having the cut site for MSRE cannot be analyzed and (2) single $\mathrm{C}$-resolution is not feasible for the assays with more than one cut sites in $\mathrm{CpG}$ and one has to assume that investigated regions are homogenously methylated, which is a common assumption in epigenetics [6]. However, using different combinations of MSREs and varying product length can adjust the number of CpGs per assay (Table 2). A restriction map can help to identify the correct MSRE (or any combination of different MSREs) and gives an overview where and how often the fragments of interest get cleaved (Table 2). In the present study, to maximize the number of CpGs covered by MSREs, a combination of four different enzymes was chosen to conduct the experiment. The total percent coverage of CpGs for these four enzymes is about $39 \%$ (AciI, 17.4 \%; Hin6I, 6.4 \%; HpaII, $8.6 \%$; and HpyCH4IV, $6.6 \%)$ of the whole human genomic DNA (Table 2) [4].

In past years, clinical epigenetic studies have shown the importance and successful utilization of the MSREPCR method [3-5, 7-11]. Despite the successful use of this method, there are no one-stop software tools available to design a large number of optimized MSRE-specific assays in a time-efficient manner in parallel. There are several software tools available to design general-purpose PCR primer pairs as well as oligos such as PerlPrimer [12], Primer3 [13], Primer3Plus [14], Primer Express [15], Primer Select [16], BatchPrimer3 [17], Primer Premier

Table 1 Comparison of different DNA methylation analysis methods

\begin{tabular}{|c|c|c|}
\hline Method & Advantages & Limitations \\
\hline \multirow[t]{4}{*}{$\mathrm{BSP} / \mathrm{MSP}$} & Highly sensitive. & Gives rise to false positivity if bisulfite modification is incomplete. \\
\hline & Highly specific for particular CpG sites. & Poor design of primers can give rise to inconclusive results. \\
\hline & $\begin{array}{l}\text { Facilitates the analysis of clinical samples with low } \\
\text { levels of methylated sequences. }\end{array}$ & Low multiplexing capabilities. \\
\hline & $\begin{array}{l}\text { Obviates the use of restriction enzymes and eliminates } \\
\text { the problem of incomplete enzyme digestion. }\end{array}$ & $\begin{array}{l}\text { Need for methylation specific probes in addition to primers - when } \\
\text { conducting BSP and hybridization probe-based distinction of ME/UM } \\
\text { sequences. }\end{array}$ \\
\hline \multirow[t]{4}{*}{$\begin{array}{l}\text { Bisulfite } \\
\text { sequencing }\end{array}$} & $\begin{array}{l}\text { Highly specific. Usually depending on BSP or MSP } \\
\text { before sequencing. }\end{array}$ & $\begin{array}{l}\text { Technically demanding; similar to MSP/BSP because depending on BSP } \\
\text { amplification. }\end{array}$ \\
\hline & $\begin{array}{l}\text { Delineates the methylation status of each individual } \\
\text { CpG site. }\end{array}$ & Time consuming and labor intensive. \\
\hline & Requires very low DNA amounts. & Gives rise to false positivity if bisulfite modification is incomplete. \\
\hline & $\begin{array}{l}\text { NGS-based high multiplexing capability when pooling } \\
\text { single-MSP-derived amplicons. }\end{array}$ & \\
\hline \multirow[t]{3}{*}{ MSRE } & $\begin{array}{l}\text { High sensitivity and enables high multiplexing and } \\
\text { quantitative read out. }\end{array}$ & $\begin{array}{l}\text { Limited to target regions, which are covered by MSRE recognition } \\
\text { sequence. }\end{array}$ \\
\hline & $\begin{array}{l}\text { Detection of low fraction }(0.1-x \%) \text { of methylated } \\
\text { in unmethylated DNA background. }\end{array}$ & Gives rise to false positivity if enzyme digestion is incomplete. \\
\hline & $\begin{array}{l}\text { Suitable for multiplexed analyses } 50-100 \text { amplicons } \\
\text { starting from ng-amounts (e.g., cfDNA). }\end{array}$ & $\begin{array}{l}\text { Internal standards recommended because no direct distinction of } \\
\text { ME/UM alleles is feasible based on the sequence (like C vs T upon } \\
\text { BS-based analysis in sequences) when aiming NGS-based readout. }\end{array}$ \\
\hline
\end{tabular}


Table 2 Whole genome MSREs coverage of CpGs in human genomic DNA [4]

\begin{tabular}{lllll}
\hline MSRE & $\begin{array}{l}\text { Recognition } \\
\text { sequence }\end{array}$ & $\begin{array}{l}\text { Percentage } \\
\text { coverage of } \\
\text { CpGs in human } \\
\text { gDNA (\%) }\end{array}$ & $\begin{array}{l}\text { Number of } \\
\text { fragments } \\
\text { (per kb) in } \\
\text { CpG islands }\end{array}$ & $\begin{array}{l}\text { Number of } \\
\text { fragments } \\
\text { (per kb) in }\end{array}$ \\
non-CpG islands
\end{tabular}

[18], PRIMEGENS [19], and PrimerBlast [20]. These tools are not optimized for methylation-specific primer design and do not support multiplexed and high-throughput primer design in a time-effective manner.

Primer3 is a widely used program for designing PCR primers and is the basis for many other tools, but does not provide genomic information about resulting primer pairs and only reports relative coordinate information to the target sequence. BatchPrimer3 is a batch primer tool, which allows use of multiple target sequences but has limitations on target length and does not design MSREPCR primers. None of the other tools provide MSREPCR design, genomic annotation (i.e., single-nucleotide polymorphism (SNP), CpG locus annotation, upstream and downstream RefSeq gene information and cut site information), and efficient and accurate primer pair selection by using a user-defined quality-filtering matrix to reduce the post processing.

PerlPrimer and PRIMEGENS perform DNA methylationbased primer design by identifying the cut sites and CpG islands, but they do not provide genome, SNP, and repeat information for filtering primer pairs. Furthermore, they do not support user-defined primer pair selection and filtering to pick the best and reliable primer pairs.

We have therefore developed MSRE-HTPrimer, an open source, web-based, and high-throughput primer design pipeline for MSRE-PCR and genomic-PCR primers capable of simultaneously processing hundreds to thousands of target sequences. To achieve that goal, we have adapted the current Primer3 primer design process and added genomic annotations, multiprocessing capabilities, and new primer selection possibilities. The setup and validation of MSREqPCR were done in accordance with the MIQE guidelines, representing the minimum information for publication of quantitative real-time PCR experiments [21] to ensure reliable experimental results.

\section{Results and discussion}

MSRE-HTPrimer is an open-source, portable, webbased, and easy-to-use pipeline, which facilitates the design of primer pairs for epigenetic and genomic target validation studies. It uses a simple input and output model and can design primers for hundreds to thousands of target sequences in a single run. Moreover, it does not have any limitations on the number and size of target sequences. MSRE-HTPrimer provides significant improvements over existing solutions with following unique features: (1) visualization of primer pairs in UCSC genome browser [22], (2) search each resulting primer pair in UCSC In-Silico PCR database [23], (3) flexible primer selection and filtering based on custom quality matrix, and (4) parallel primer design for several target sequences. The pipeline is equipped with multiprocessing capability and uses custom inputs and parameters to design specific primers. All components of MSRE-HTPrimer pipeline workflow, inputs, and outputs have been summarized in Fig. 1.

\section{MSRE-HTPrimer pipeline}

The MSRE-HTPrimer pipeline (Fig. 1) consists of seven sequential steps. Based on a user-defined list of target regions and design parameters, the pipeline retrieves a list of annotated primer pairs (in TEXT and HTML format) and links to visualize results in UCSC genome browser and UCSC In-Silico PCR. All steps of the pipeline are described as following:

1. Download and prepare reference sequence and annotation from UCSC genome browser After installation, when the user starts the first primer design process, MSRE-HTPrimer downloads and prepares the reference FASTA sequence, common SNPs, RefSeq gene, CpG islands, and known repeat elements annotation for the entire genome (human and mouse) based on the selected genome, genome assembly, and dbSNP build number from the UCSC genome browser. The default genome on query page is human, genome assembly is hg19, and the dbSNP build number is 142. MSRE-HTPrimer does not re-download reference data for subsequent primer design if it is already downloaded and prepared. From the query interface, the user can customize all primer design and selection parameters for both MSRE-PCR and genomic-PCR.

2. Define primer design range for each target region In this step, the primer design genomic range is prepared by adding the number of flanking upstream and downstream base pairs (optional) to the actual target region given as input in the target bed file (Additional file 1).

3. Prepare FASTA sequence for each target region 


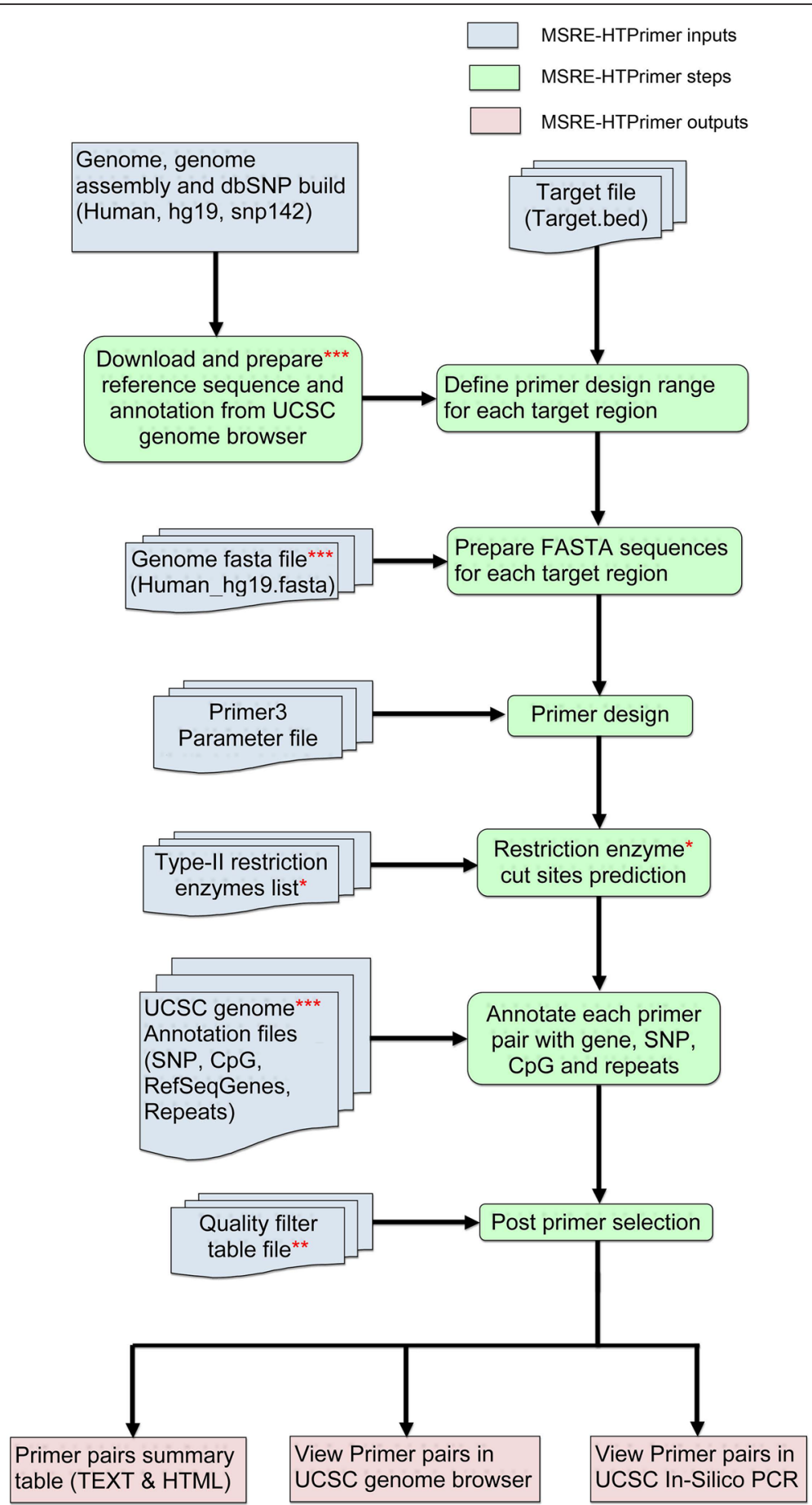

Fig. 1 The workflow of MSRE-HTPrimer pipeline. The MSRE-HTPrimer pipeline can be run via an intuitive web interface. The sequential analysis steps are displayed from top to bottom. (Single asterisk) Restriction enzyme cut sites prediction step and the Type-ll restriction enzymes list input are only applicable for MSRE-PCR. (Double asterisks) Quality filter table file is optional; if user-defined primer selection criteria are not provided, then all primer pairs will be recorded in the final output summary table. (Triple asterisks) Download and prepare reference sequence and annotation from UCSC genome browser step runs only once first time or when reference genome information is changed

Next, target FASTA sequences are extracting from the genome reference using the target regions from step 2. The genome reference FASTA file and common SNPs, RefSeq gene, CpG islands, and "repeats-annotation" files for human or mouse are downloaded and prepared from the UCSC genome 
browser in step 1. In this step, MSRE-HTPrimer also subset the large annotation files based on target regions coordinates using BedTools [24], which makes execution faster.

4. Primer design with Primer3

In this step, the tool takes two inputs: (1) FASTA sequences from the previous step and (2) a Primer3 parameter input file (Additional file 2). It runs the Primer3 tool [25] to design all possible PCR primer pairs and oligos (optional) for all targets sequences and stores all resulting primers, oligos, and amplicons.

5. Restriction enzyme cut sites prediction In this step, the enzymes cut sites in forward primers, reverse primers, oligos, and amplicons are calculated. This step is only applicable for MSRE-PCR primer design; optionally, when users design genomic PCR primers, this step can be omitted.

6. Annotate each primer pair with gene, SNP, CpG, RefSeq genes, and repeats

In this step, each primer pair, oligo, and amplicon is annotated with RefSeq genes found upstream and downstream of the target region, SNPs, CpG islands, and repeat regions. These annotations will help to pick the accurate and sensitive primer pairs for each target region.

7. Post primer selection

Based on the user-defined selection criteria, the final primer pairs for each target region are selected. These selection criteria can be given as and input file which is specified in Additional file 3. This step facilitates selection of primer candidates and provides the specific primer pairs for hundreds to thousands of target regions in a time-effective manner. This hierarchical filtering process is a unique and very useful feature of the MSRE-HTPrimer, which is lacking in all other similar available tools. Finally, MSRE-HTPrimer produces primer summary table in TEXT and HTML format and visualizes results in UCSC genome browser [22] and UCSC In-Silico PCR database [23].

\section{Query interface}

MSRE-HTPrimer offers a very intuitive, user-friendly, and powerful query interface (Fig. 2a). MSRE-HTPrimer allows the user to design genome-wide primers for any number of target sequence in a single run. The user can select the appropriate genome name, genome assembly, and the dbSNP build as per requirements. For primer design, the user can upload a target bed file, Primer3 parameter file, type-II enzyme list for MSRE-PCR primer, and custom primer selection matrix file. Moreover, the user can customize several primer design and selection parameters to obtain specific and optimized primer pairs and product. The query page can be opened with http://localhost/msre-htprimer page.

\section{MSRE-HTPrimer input}

MSRE-HTPrimer requires four input files:

1. Target BED file: This file contains the genomic coordinates for all target sequences (one line for each target sequence). It consists of four tab-delimited columns: (1) chromosome, (2) start coordinate, (3) end coordinate, and (4) a unique ID for each target region (Additional file 1).

2. Primer3 parameter file: This text file contains the parameters and values for the Primer3 tool. It is optional and if not provided, MSRE-HTPrimer will use default Primer3 parameters (Additional file 2)

3. Restriction enzyme file: This input file is only required for MSRE-PCR primer design. Each line contains an enzyme name as per nomenclature, and multiple enzymes are allowed in a single run (Additional file 4).

4. Custom primer selection quality matrix.

MSRE-HTPrimer supports selection of primer pairs based on user-defined selection criteria. A custom qualityfiltering matrix can be provided as input file. As shown in Additional file 3, the user can define a set of selection criteria and rank them using a scale of 1-10. MSREHTPrimer assigns these ranks to the primer pairs for all target sequences. If this input is not provided, then primer pairs are returned based on Primer3 ranking. MSREHTPrimer supports mathematical operators, including ">," " $<$," " >=," " $<=$," and "-". Any column header of the MSREHTPrimer output file can be used as parameter. The primer quality level represents the hierarchical rank associated with each of the output parameters in its respective row.

\section{MSRE-HTPrimer output}

MSRE-HTPrimer produces a summary output file in two formats: (1) a tab delimited text file and (2) HTML output file (See Additional file 5 and Fig. 2b), which contains one line for each primer pair along with all annotations including target sequence ID, amplicon ID, oligos and genome amplicon coordinates, number of cut sites, number of SNPs, number of CpG islands, repeat regions, upstream and downstream RefSeq genes and their distance with respect to forward and reverse primer, and direct link to UCSC genome browser and UCSC Insilco-PCR. For both primer design methods (MSREPCR and Genomic-PCR), it produces a uniform output, which facilitates an easy output handling, post processing, and management. The HTML summary output table has a direct hyperlink to UCSC genome browser and UCSC In-Silico PCR database.

\section{Visualization of primer pairs}

MSRE-HTPrimer offers the visual display of resulting primer pairs in UCSC genome browser along with genomic 

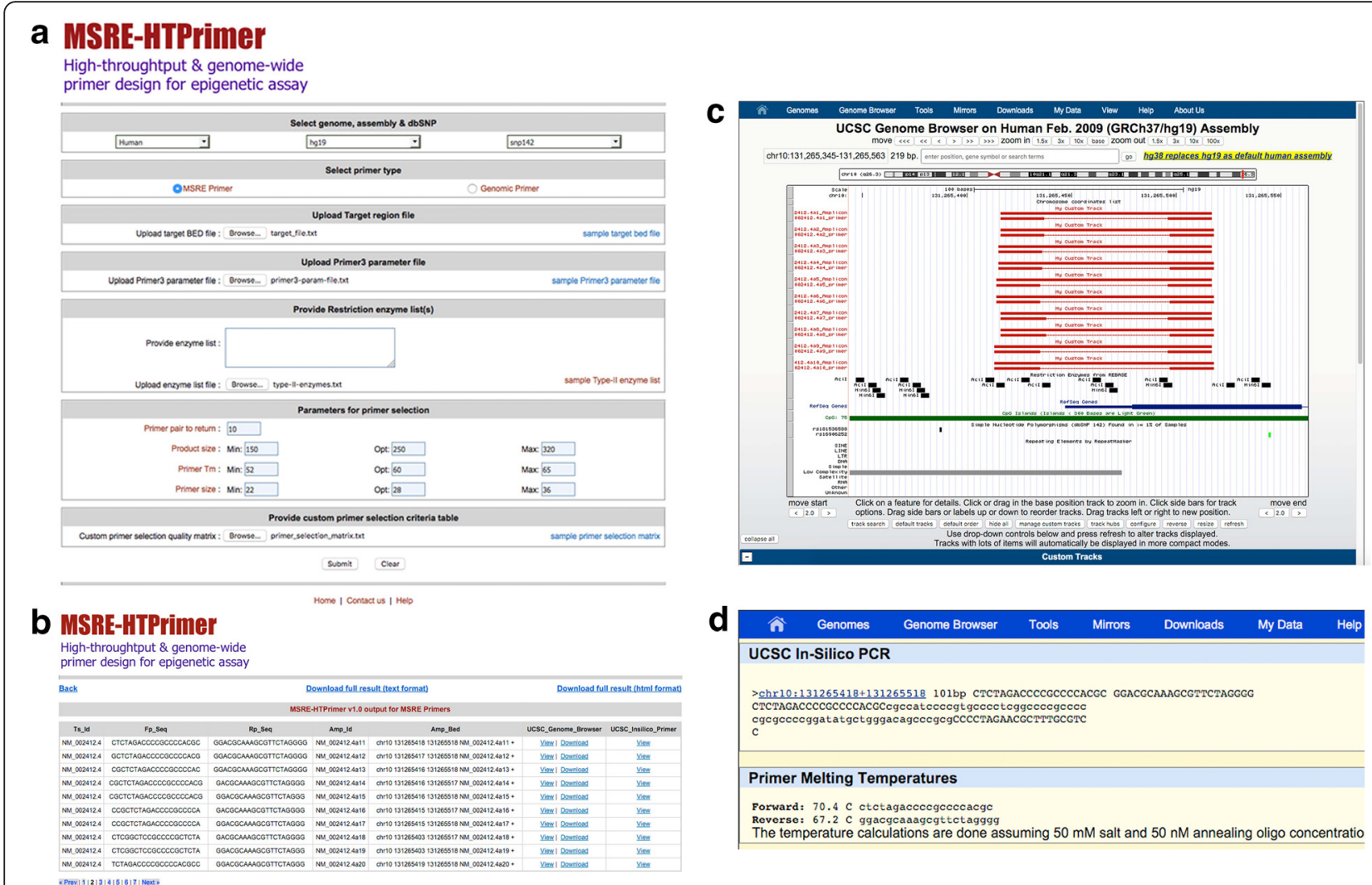

UCSC In-Silico PCR

>chr10:131265418+131265518 $101 \mathrm{bp}$ CrCPACACCCCCCCCCACOC GGACCCAAACCGMTCPACGCG

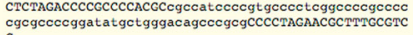

\section{Primer Melting Temperatures}

Forward: 70.4 c ctctagaccecgccccacgc
Reverse: 67.2 C gaacgcaaagcgttctagggg

The temperature calculations are done assuming $50 \mathrm{mM}$ salt and $50 \mathrm{nM}$ annealing oligo concentratio

Fig. 2 The MSRE-HTPrimer web interface and output. a Web interface of MSRE-HTPrimer query page (the query interface for MSRE-HTPrimer shows different parameters that can be used to design and select optimized PCR primers for MSRE assay and genomic sequencing assay). $\mathbf{b}$ An example of primer pair summary output table in MSRE-HTPrimer web interface (an example of primer pair summary table for RefSeq Human gene MGMT [chr10: 129467190-129768042, RefSeq ID NM_002412.4]. It contains target sequence ID, forward and reverse primer sequence, amplicon coordinates in BED format, link to display and download to UCSC genome browser, and display to In-Silico PCR database). c An example output of the MSRE-HTPrimer primer pair visualization in UCSC genome browser along with genomic (MSRE cut sites, RefSeq, CpG islands, SNPS, and Repeats) track within MSRE-HTPrimer web interface (in order to focus and illustrate MSRE-HTPrimer features, all redundant UCSC genome browser tracks are hidden in 2 C presenting from top to bottom; the MSRE cutsites position, the localization of RefSeq Human gene MGMT [chr10: 129467190129768042, RefSeq ID NM_002412.4], CpG islands, simple nucleotide polymorphisms based on dbSNP 142 [rs18153536588 and rs16906252], and the kind of repeating elements [low complexity]. Each result of the primer design pipeline is presented bundled, once as single red line [full amplicon] and as a line emphasizing forward and reverse primer below). $\mathbf{d}$ An example output of In-Silico PCR database search display of primer pairs (an example of primer pair display in UCSC In-Silico PCR database for RefSeq human gene MGMT [chr10: 129467190-129768042, RefSeq ID NM_002412.4])

annotations, CpG islands, common SNPs, RefSeq genes, restriction enzymes and other genomic information (Fig. 2c), and in UCSC In-Silico PCR database (Fig. 2d). In addition, MSRE-HTPrimer also provides the primer pairs for each target sequence as a UCSC custom track file in GTF format (Additional file 6), which can be used for other analysis or can be visualized in other genome browser. The custom track bed file is created for each target sequence and can be downloaded from the summary output table (see Fig. 2b).

\section{Availability, installation, and usage}

MSRE-HTPrimer is a stand alone, portable, and webbased pipeline, freely available for all researchers. It is available as a fully configured Virtual Machine accessible at http://sourceforge.net/p/msrehtprimer/wiki/Virtual_
Machine/. An extensive user manual is available at https://sourceforge.net/projects/msrehtprimer/files/Manual. pdf. A test data set is available at https://sourceforge.net/ projects/msrehtprimer/files/test_data.zip.

\section{Performance evaluation}

MSRE-HTPrimer is a high-throughput primer design pipeline and has no restriction either on the number of assays or on the size of target sequences. To evaluate the performance of MSRE-HTPrimer, we have randomly selected 200 target sequences of different lengths from human gene ESR1-estrogen receptor 1 (chr6:152011631152424408) and executed the benchmarking on a Linux server (Ubuntu 12.0.4 LTS with 8 CPU, 16 GB RAM). Execution times were measured for both MSRE-PCR (black line) and genomic-PCR (red line). All benchmark 
measurements have been performed using the Primer3 version 2.3.6 focusing on different sizes and number of target sequences in relation to runtime. All execution times were measured in seconds. As shown in Fig. 3a, b, MSRE-HTPrimer is very fast and efficient to design specific primer pairs for hundreds of target regions. As shown, design for 100 MSRE-PCR assays is conducted in less than $1500 \mathrm{~s}$ (25 $\mathrm{min}$ ) computing time to run the entire steps according to the pipeline. For the same dataset,
MSRE-PCR design takes more time than genomic-PCR design (e.g., $<500 \mathrm{~s}$ designing 100 assays), which is due to restriction enzyme cut site annotation (Fig. 3b).

\section{MSRE-HTPrimer experimental validation}

We have experimentally validated the primers designed by our MSRE-HTPrimer pipeline for methylation sites selected from a genome-wide discovery study. Predefined design and filtering parameters of the MSRE-HTPrimer

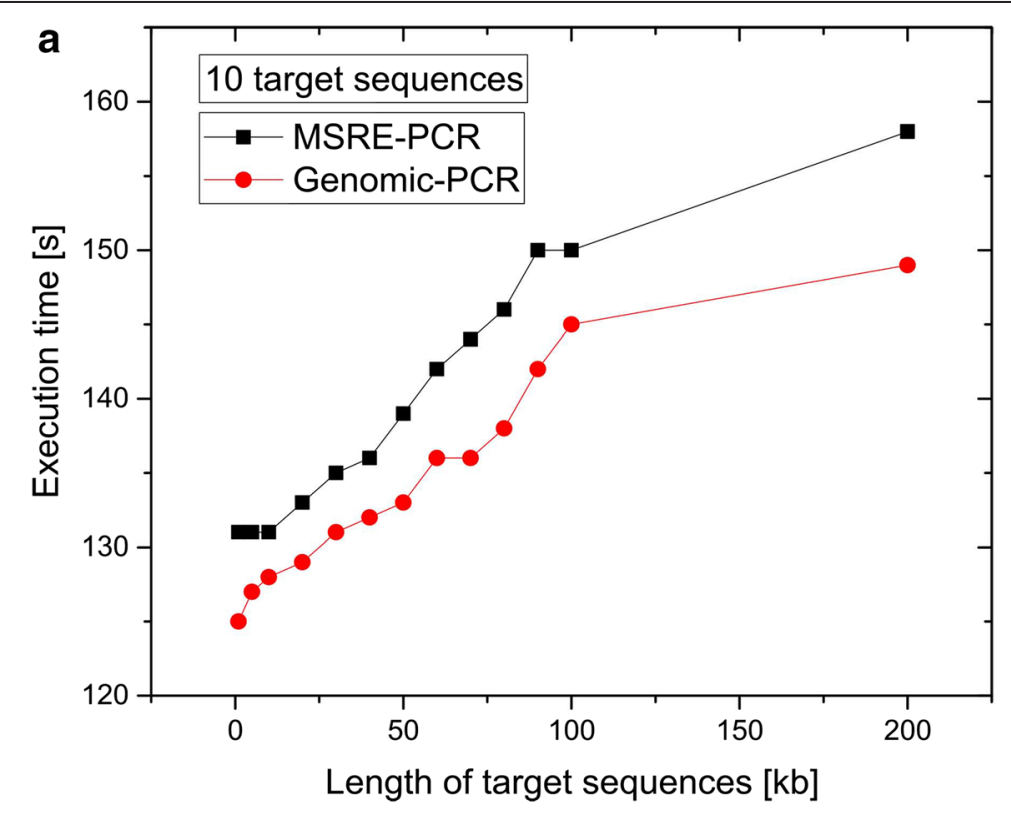

b

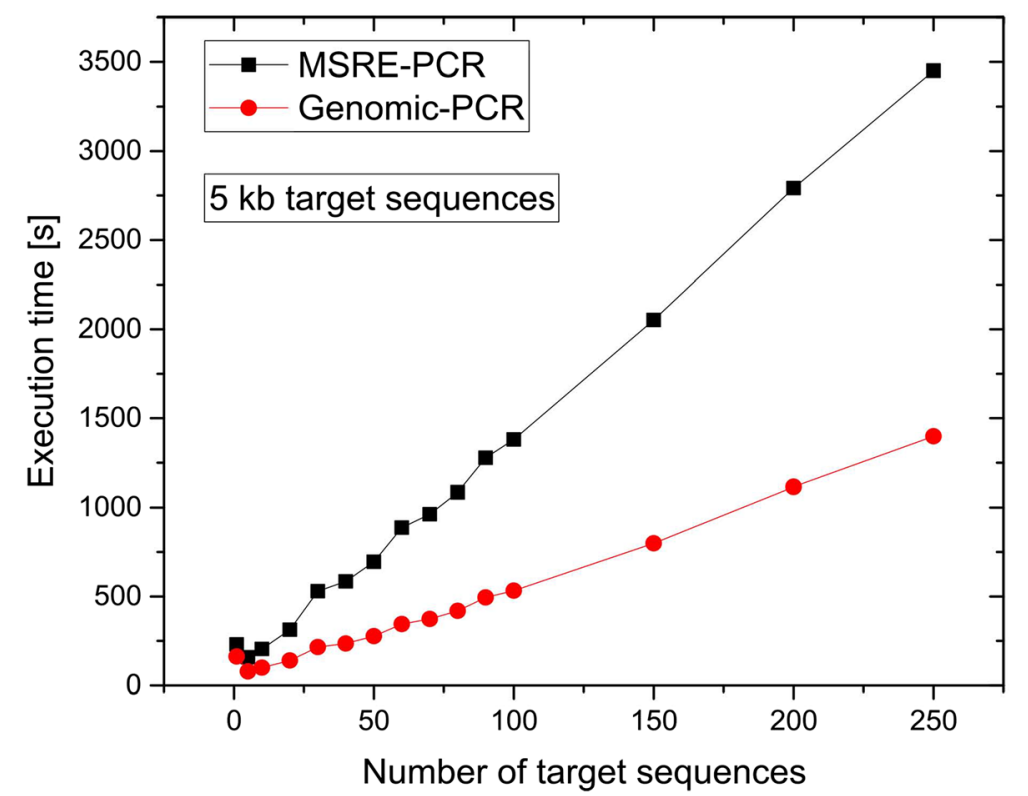

Fig. 3 Evaluation of MSRE-HTPrimer execution for MSRE-PCR (black line) and genomic-PCR (red line), considering the number and size of target sequences. a Different sizes of target sequences $(1,5,10,20,30,40,50,60,70,80,90,100$, and $200 \mathrm{~KB})$, each time using 10 target sequences of the same length. $\mathbf{b}$ Different numbers of target sequences $(1,5,10,20,30,40,50,60,70,80,90,100,150,200$, and 250 sequences of equal length of $5 \mathrm{~kb})$ 
tool, such as the amount of cut sites per assay, position of the cut sites, SNP filtering, and avoiding position with repeats, combined with the standard parameters of Primer3 (e.g., sequence length, melting temperature, GC content, and primer length) yielded a total of 190 MSREqPCR assays. Running those under the same PCR conditions, out of 190 primer pairs, 135 (71.05\%) were qualified for qPCR according MIQE guidelines. Details on performance parameters can be found in the Additional file 7. The validation results are shown in Fig. 4 and include the performance parameters efficiency (deduced from slope by the formula $E=\left(10^{(-1 / \text { slope })}-1\right) * 100$; average efficiency of 135 assays, $92.03 \%$ ), the correlation coefficient (average $R^{2}, 0.993$ ) and the theoretical $1 \mathrm{ng}$ detection (average ct: 29.11 ) of the assays, which were deduced from the calibration curve. These results are in line with our experiences when qualifying "manually" designed sets of assays and also comparable to MSP as well as BSP-based assays where our internal success rate is between 70 and $80 \%$ (data not shown).

\section{Intended user groups}

The major advantage of the MSRE-HTPrimer pipeline in contrast to other bioinformatics primer design tools is its flexibility and multiprocessing capability to design primers for thousands of targets in parallel. Moreover, it decreases the post-processing steps by applying efficient filtering and providing selection criteria based on a userdefined quality-filtering matrix. It allows users to develop optimized assays and thus significantly increases speed and success rate of genetic as well as epigenetic primers design for validation. The indented user group includes researchers working in the genetic and epigenetic domain. The genomic-PCR method of MSRE-HTPrimer adds another level of information to the primer design by giving researchers the possibility to design primer pairs based on specific genomic traits or locations (SNP, RefSeq, CpG islands, repeats). Furthermore, the MSRE design approach enables the design of primer pairs specifically for the analysis of DNA methylation or for bisulfite deamination-based MSP.

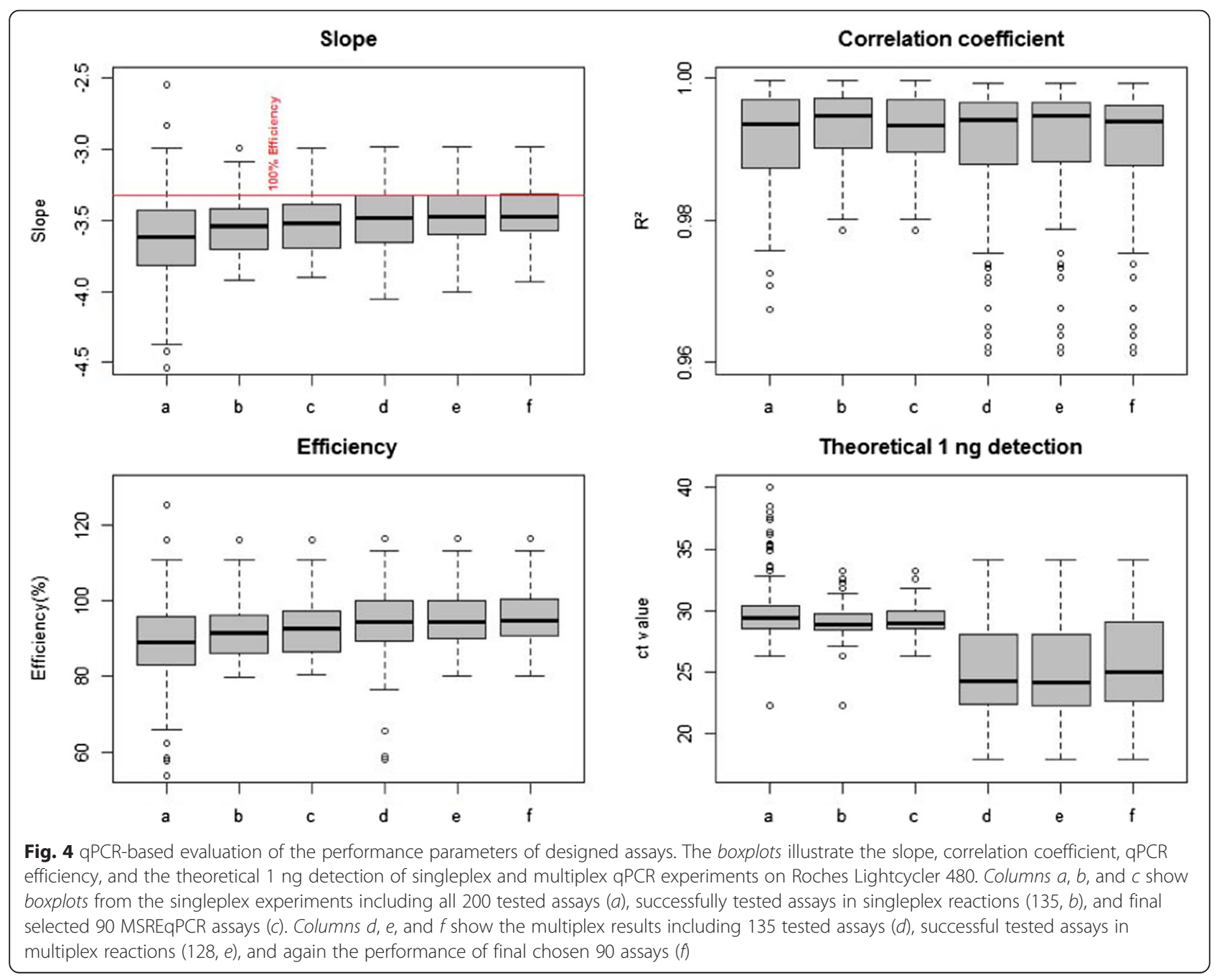




\section{Comparison with existing tools}

Today, several open source and commercial primer design software/tools are available including, PerlPrimer, Primer3, Primer3Plus, PrimerSelect, Batchprimer3, PrimerPremier, PRIMEGENS, and PrimerBlast. The comparison of the MSRE-HTPrimer pipeline to each of these tools is shown in Table 3. In total, 19 different points including availability, operating system, and installation requirements as well as necessary dependencies, multiprocessing capabilities, limitations of input file size, target sequence length, amount of target sequences, visualization of results in UCSC genome browser, and genomic annotation of results were evaluated. MSRE-HTPrimer is available for free and can be run on any Unix system. Furthermore, it is available as a fully configured virtual machine. MSREHTPrimer has no limitations concerning the input file size, the amount of target sequences, or the target sequence length. Moreover, it is capable of multiprocessing the target sequences reducing the time for highthroughput primer design. In addition, MSRE-HTPrimer provides many advance features over existing tools such as (1) assigning genomic coordinates to all resulting primer pairs, oligos, and amplicons; (2) annotate all resulting primers with SNPs, RefSeq genes, repeat elements and CPG islands and automatic FASTA sequence preparation based on input target BED file (Additional file 1); (3) positioning of restriction enzyme cut sites accurately; and (4) visualization of results in the, e.g., UCSC genome browser. Finally, MSRE-HTPrimer is extremely useful for methylation-sensitive restriction enzyme-based PCR and any genetic primer design using non-bisulfite deaminated DNA sequences.

\section{Conclusions}

We provide MSRE-HTPrimer, a robust, user-friendly, web-based, standalone, one-stop high-throughput, and genome-wide epigenetic primer design pipeline with multiprocessing capabilities. MSRE-HTPrimer annotates all resulting primer pairs extensively by adding genetic and epigenetic information including SNPs, RefSeq genes, repeats, and $\mathrm{CpG}$ islands. It enables primer design for thousands of target sequences in a single run with great accuracy and greatly facilitates post processing. MSREHTPrimer has no limitation on the number and size of target sequences and provides full flexibility to customize the Primer3 parameters for each specific requirement. Furthermore, it offers the opportunity to rank primer pairs based on task-specific preferences using a custom quality filter matrix in addition to general Primer3 ranking. In comparison to other tools, MSRE-HTPrimer stands out

Table 3 Comparison of various features of MSRE-HTPrimer and other tools for primer design

\begin{tabular}{|c|c|c|c|c|c|c|c|}
\hline Features & $\begin{array}{l}\text { MSRE- } \\
\text { HTPrimer v1.0 }\end{array}$ & $\begin{array}{l}\text { Primer } \\
\text { Express v3.0.1 }\end{array}$ & $\begin{array}{l}\text { Primer } \\
\text { Select }\end{array}$ & $\begin{array}{l}\text { Primer } \\
\text { Premier }\end{array}$ & $\begin{array}{l}\text { Perl } \\
\text { Primer v1.1.21 }\end{array}$ & $\begin{array}{l}\text { PRIMEGENS- } \\
\text { V2.0 }\end{array}$ & BiSearch \\
\hline Genome-wide primer design & Yes & No & No & Yes & No & No & No \\
\hline Dependency & No & Yes & Yes & Yes & Yes & Yes & Yes \\
\hline Installation required & No & Yes & Yes & Yes & Yes & Yes & No \\
\hline Operating system & $\begin{array}{l}\text { All Unix, MAC, } \\
\text { Windows }\end{array}$ & Windows & - & $\begin{array}{l}\text { Windows, } \\
\text { MAC }\end{array}$ & $\begin{array}{l}\text { All Unix, MAC, } \\
\text { Windows }\end{array}$ & $\begin{array}{l}\text { All Unix, } \\
\text { Windows }\end{array}$ & Web \\
\hline Genome coordinate information ${ }^{a}$ & Yes & No & No & No & No & No & No \\
\hline SNP annotation ${ }^{a}$ & Yes & No & No & Yes & No & No & No \\
\hline Repeat element annotation ${ }^{a}$ & Yes & No & No & Yes & No & No & No \\
\hline CpG Islands annotation ${ }^{a}$ & Yes & No & No & No & Yes & No & No \\
\hline RefSeq gene annotation ${ }^{a}$ & Yes & No & No & Yes & No & No & No \\
\hline Restriction enzyme type-\| cut sites identification ${ }^{a}$ & Yes & No & No & No & No & No & No \\
\hline Multi-processing capability & Yes & Yes & No & Yes & No & Yes & No \\
\hline Multiple target sequences & Yes & Yes & No & Yes & No & Yes & No \\
\hline Target sequence number restriction ${ }^{a}$ & No & Yes & Yes & Yes & Yes & Yes & Yes \\
\hline Target sequence length restriction ${ }^{a}$ & No & Yes & Yes & Yes & Yes & Yes & Yes \\
\hline FASTA sequence selection by tool & Yes & No & No & Yes & Yes & No & No \\
\hline Custom primer selection quality matrix ${ }^{a}$ & Yes & No & No & No & No & No & No \\
\hline Input file limitations & No & No & No & No & No & No & No \\
\hline UCSC genome browser visualization ${ }^{a}$ & Yes & No & No & No & No & No & No \\
\hline UCSC In-Silico primer design page cross-link & Yes & No & No & No & No & No & No \\
\hline Availability & Free & Commercial & Commercial & Commercial & Free & Free & Free \\
\hline
\end{tabular}

${ }^{\mathrm{a}}$ Denotes the unique feature of MSRE-HTPrimer in comparison to the other tools 
for high-throughput, genome-wide, and optimized epigenetic primer design capability, improved primer design accuracy, efficient primer selection, and primer visualization in UCSC genome browser.

\section{Methods}

\section{Pipeline development}

The MSRE-HTPrimer pipeline was developed using Python 2.7.10 (http://www.python.org) and Biopython (http://biopython.org) with special focus on multiprocessing capability to design two types of primers: (1) epigenetic primers (MSRE-PCR) and (2) genomic and sequencing primers (genomic-PCR) with great efficiency and success rate in a high-throughput manner. The reference genome FASTA sequence and annotations are used from UCSC genome browser, which are automatically retrieved and prepared by MSRE-HTPrimer. The minimum user-defined input requirements to run MSRE-HTPrimer are (1) target genomic regions in BED format file, (2) type-II enzyme(s) for MSRE primers, (3) genome name, assembly, and dbSNP version. The MSRE-HTPrimer workflow is depicted in Fig. 1 consisting of seven sequential steps and starts with an arbitrary list of target regions and outputs a list of annotated primer pairs.

\section{Web interface development}

The MSRE-HTPrimer web interface was developed using the HTML, Perl, and CGI and runs on an Apache web server. The graphical display of designed primer pairs and products for all target sequences are visualized in the UCSC genome browser and the UCSC In-Silico PCR database. Hence, MSRE-HTPrimer uniquely depicts the designed primer pairs in the UCSC genome browser along with genomic annotation, restriction enzymes, repeats, conservation, RefSeq genes, and other information available in UCSC genome browser.

An extensive user manual (Additional file 8) is provided including description of inputs, parameters, outputs, installation dependencies, MSRE-HTPrimer usage, and a detailed step-by-step description of the MSREHTPrimer pipeline. The tool along with intuitive web interface is available as a fully configured Virtual Machine (VM), which can be run using the Virtual box system (https://www.virtualbox.org/). The virtual machine is configured to run without any installation and configuration needs.

\section{Experimental validation of MSRE-HTPrimer}

An epigenome-wide experiment using DNA methylation arrays from Illumina (Infinium HumanMethylation450 Bead Chips, Illumina, California, USA) was used to define regions for assay design and to further experimentally qualify the PCR primers designed by the MSREHTPrimer pipeline in the lab. The Infinium 450k assay provides distinct information about the methylation level of 485,577 cytosine sites per sample at single-nucleotide resolution within the human genome. Based on statistical analysis of the $450 \mathrm{k}$ data as well as other "biological" criteria (data not shown), a panel of 190 target regions referring to corresponding $\mathrm{CpG}$ sites presented by the 450k array (Additional file 7 and Additional file 9) were selected for methylation analyses by MSRE-PCR. The MSRE-PCR design parameters were set as following: the PCR product must have at least one restriction enzyme cut site ideally within the amplicon as well as no SNP within the primer sequence and no common repeats within the assay. Furthermore, each original target position has to be situated inside or close $( \pm 50 \mathrm{bp})$ to the PCR sequence. MIQE conform performance parameters of the designed assays was evaluated by qPCR using a Roche Light Cycler 480 system. Therefore, DNA from peripheral blood was serially diluted and applied to the qPCR reactions to create a calibration curve with four calibration points (10 ng/reaction; $2.5 \mathrm{ng} /$ reaction; $0.625 \mathrm{ng} /$ reaction; $0.156 \mathrm{ng} /$ reaction-dilution-factor 4).

The performance parameters were directly deduced from the calibration curve: A slope of -3.32 correlates with $100 \%$ qPCR efficiency and indicates a doubling of the strands during PCR. The theoretical $1 \mathrm{ng}$ detection gives information of the expected cp-values when only $1 \mathrm{ng}$ of DNA is applied to the qPCR reaction and is directly deduced from the intersection with the $y$-axis (intercept). Performance of the assays was evaluated in single-plex reactions as well as in multiplex reaction. For the multiplex reaction, a pre-amplification containing all 135 primer pairs was executed on a conventional PCR cycler; subsequently, the pre-amplified targets were analyzed by Roche's Light Cycler 480.

\section{Additional files}

Additional file 1: Target bed file (mandatory input file) to run primer design with MSRE-HTPrimer. (TXT 256 bytes)

Additional file 2: Primer3 parameter input file. This is the parameter for Primer3 tools to design primer pairs. Users can change these parameters to optimize and better positioning of the primer pairs for target region. These parameters are directly supplied to Primer3 tool. (TXT 477 bytes)

Additional file 3: Custom quality filter matrix with ten quality levels ranking the designed primer independent of the Primer3 level, but dependent on amplicon size, amount of cut sites, and gene distance. (TXT 870 bytes)

Additional file 4: List of restriction enzymes (mandatory input for MSRE-PCR) in a text file one enzyme per line. (TXT 30 bytes)

Additional file 5: MSRE-HTPrimer output summary table. MSRE-HTPrimer produces a final output summary with one line per primer pairs including target sequence information, primer pairs, oligos, amplicons, and their genomic and epigenetic annotations. (XLS $234 \mathrm{~kb}$ )

Additional file 6: An example output UCSC custom track in GTF format produced by MSRE-HTPrimer for each target sequence in a separate file. (GTF $3 \mathrm{~kb}$ ) 
Additional file 7: Detail description of 135 experimentally validated MSREqPCR assays designed by MSRE-HTPrimer. (XLSX 57 kb)

Additional file 8: MSRE-HTPrimer user manual. An extensive guide for user to design primers with MSRE-PCR and genomic-PCR methods. (PDF 7698 kb)

Additional file 9: The target bed file, which contains 190 target regions, used for experimental validation. (TXT $7 \mathrm{~kb}$ )

\section{Competing interests}

The authors declare that they have no competing interests.

\section{Authors' contributions}

AW, AK, and RVP designed the study. RVP developed the algorithms and wrote the software. RK, GB, PW, and AW performed the experimental validation. RVP, PW and RK wrote the manuscript. AW and SP revised the manuscript. All authors have revised the manuscript and approved the final version.

\section{Acknowledgements}

We thank all members of the Health \& Environment Department, Molecular Diagnostics, AIT_Austrian Institute of Technology GmbH, Vienna, Austria for the testing of MSRE-HTPrimer, feedback, and useful comments. Part of this work was supported by: European Community's Seventh Framework program (FP7/2007-2013) EurHEALTH Ageing HEALTH-F2-2011-277849.

\section{Author details}

${ }^{1}$ Health \& Environment Department, Molecular Diagnostics, AIT-Austrian Institute of Technology $\mathrm{GmbH}$, Vienna, Austria. ${ }^{2}$ Institut für

Populationsgenetik, Vetmeduni Vienna, Veterinärplatz 1, 1210 Vienna, Austria.

Received: 29 August 2015 Accepted: 23 February 2016

Published: 5 March 2016

\section{References}

1. Goldberg AD, Allis CD, Bernstein E. Epigenetics: a landscape takes shape Cell. 2007;128(4):635-8.

2. Costello JF, Plass C. Methylation matters. J Med Genet. 2001;38:285-303.

3. Melnikov AA, Gartenhaus RB, Levenson AS, Motchoulskaia NA, Levenson W. MSRE-PCR for analysis of gene-specific DNA methylation. Nucleic Acids Research. 2005; 33(10), doi:10.1093/nar/gni092.

4. Schumacher A, Kapranov P, Kaminsky Z, Flanagan J, Assadzadeh A, Yau P, et al. Microarray-based DNA methylation profiling: technology and applications. Nucleic Acids Res. 2006;34(2):528-42.

5. Wielscher M, Vierlinger K, Kegler U, Ziesche R, Gsur A, Weinhäusel A. Diagnostic performance of plasma DNA methylation profiles in lung cancer. Pulmonary Fibrosis and COPD EBioMedicine. 2015;2(8):927-34.

6. Huynh JL, Garg P, Thin TH, Yoo S, Dutta R, Trapp BD, et al. Epigenome-wide differences in pathology-free regions of multiple sclerosis-affected brains. Nat Neurosci. 2014;17(1):121-30.

7. Pulverer W, Hofner M, Preusser M, Dirnberger E, Hainfellner JA, Weinhaeusel A. A simple quantitative diagnostic alternative for MGMT DNA-methylation testing on RCL2 fixed paraffin embedded tumors using restriction coupled qPCR. Clin Neuropathol. 2014:33(1):50-60.

8. Weinhaeusel A, Thiele S, Hofner M, Hiort O, Noehammer C. PCR-based analysis of differentially methylated regions of GNAS enables convenient diagnostic testing of pseudohypoparathyroidism type Ib. Clin Chem. 2008:54(9):1537-45.

9. Schumacher A, Weinhäusl A, Petronis A. Application of microarrays for DNA methylation profiling. Methods Mol Biol. 2008;439:109-29.

10. Olkhov-Mitsel E, Bapat B. Strategies for discovery and validation of methylated and hydroxymethylated DNA biomarkers. Cancer Med. 2012;1(2):237-60.

11. Egger $G$, Wielscher $M$, Pulverer W, Kriegner A, Weinhäusel A. DNA methylation testing and marker validation using PCR: diagnostic applications. Expert Rev Mol Diagn. 2012;12(1):75-92.

12. Marshall OJ. PerlPrimer: cross-platform, graphical primer design for standard, bisulphite and real-time PCR. Bioinformatics. 2004;20(15):2471-2.

13. Steve Rozen, kaletsky HJ Primer3. 1998. Web site http://biotools.umassmed. edu/bioapps/primer3_www.cgi.
14. Untergasser A, Nijveen $H$, Rao X, Bisseling $T$, Geurts R, Leunissen JAM. Primer3Plus, an enhanced web interface to Primer3. Nucl Acids Res. 2007;35:W71-5.

15. Applied Biosystems. Primer Express ${ }^{\circledR}$ Software Version 3.0 Getting Started Guide. 2004.

16. Plasterer TN. PRIMERSELECT. Primer and probe design. Methods Mol Biol. 1997:70:291-302.

17. You FM, Huo N, Gu YQ, Luo M-C, Ma Y, Hane D, et al. BatchPrimer3: a high throughput web application for PCR and sequencing primer design. BMC Bioinformatics. 2008:9:253. doi:10.1186/1471-2105-9-253

18. Primer Premier. Web site http://www.premierbiosoft.com/primerdesign/ index.html.

19. Srivastava GP, Guo J, Shi H, Xu D. PRIMEGENS-v2: genome-wide primer design for analyzing DNA methylation patterns of $\mathrm{CpG}$ islands. Bioinformatics. 2008;24(17):1837-42.

20. Ye J, Coulouris G, Zaretskaya I, Cutcutache I, Rozen S. Madden T Primer-BLAST: a tool to design target-specific primers for polymerase chain reaction. BMC Bioinformatics. 2012;13:134

21. Bustin SA, Benes V, Garson JA, Hellemans J, Huggett J, Kubista M, et al. The MIQE guidelines: minimum information for publication of quantitative real-time PCR experiments. Clin Chem. 2009;55(4):611-22.

22. Kent WJ, Sugnet CW, Furey TS, Roskin KM, Pringle TH, Zahler AM, et al. UCSC Genome Browser: the human genome browser at UCSC. Genome Res. 2002:12(6):996-1006.

23. In-Silico PCR: In-Silico PCR searches a sequence database with a pair of $P C R$ primers, using an indexing strategy for fast performance. Web site. https://genome.ucsc.edu/cgi-bin/hgPcr.

24. BedTools. Web site http://bedtools.readthedocs.org.

25. Untergasser A, Cutcutache I, Koressaar T, Ye J, Faircloth BC, Remm M, et al. Primer3-new capabilities and interfaces. Nucleic Acids Res. 2012;40(15):e115.

\section{Submit your next manuscript to BioMed Central and we will help you at every step:}

- We accept pre-submission inquiries

- Our selector tool helps you to find the most relevant journal

- We provide round the clock customer support

- Convenient online submission

- Thorough peer review

- Inclusion in PubMed and all major indexing services

- Maximum visibility for your research

Submit your manuscript at www.biomedcentral.com/submit 